\title{
Brief Report: Eye Movements During Visual Search Tasks Indicate Enhanced Stimulus Discriminability in Subjects with PDD
}

\author{
Chantal Kemner · Lizet van Ewijk · \\ Herman van Engeland · Ignace Hooge
}

Published online: 3 July 2007

(C) Springer Science+Business Media, LLC 2007

\begin{abstract}
Subjects with PDD excel on certain visuospatial tasks, amongst which visual search tasks, and this has been attributed to enhanced perceptual discrimination. However, an alternative explanation is that subjects with PDD show a different, more effective search strategy. The present study aimed to test both hypotheses, by measuring eye movements during visual search tasks in high functioning adult men with PDD and a control group. Subjects with PDD were significantly faster than controls in these tasks, replicating earlier findings in children. Eye movement data showed that subjects with PDD made fewer eye movements than controls. No evidence was found for a different search strategy between the groups. The data indicate an enhanced ability to discriminate between stimulus elements in PDD.
\end{abstract}

Keywords Search task - Perception - Eye movement . Eye fixation · PDD

C. Kemner $(\varangle) \cdot$ L. van Ewijk · H. van Engeland Department of Child and Adolescent Psychiatry, Rudolf Magnus Institute of Neuroscience, University Medical Center Utrecht, Heidelberglaan 100, 3584 CX Utrecht, The Netherlands e-mail: C.Kemner@umcutrecht.nl

\section{Kemner}

Department of Neurocognition, Faculty of Psychology, Maastricht University, Maastricht, The Netherlands

Present Address:

L. van Ewijk

School of Psychology and Clinical Language Sciences, University of Reading, Reading, UK

I. Hooge

Experimental Psychology, Helmholtz Instituut, Utrecht University, Utrecht, The Netherlands

\section{Introduction}

While most diagnostic criteria of Pervasive Developmental Disorder (PDD) are characterised by impairments, mostly with respect to social and communicational abilities, subjects with PDD excel on certain visuo-spatial tasks that supposedly reflect superior processing of fine detail (see Dakin \& Frith, 2005, for a review). An especially robust finding seems to be the behavior of subjects with PDD on visual search tasks (Plaisted, O'Riordan, \& Baron-Cohen, 1998; Plaisted, Saksida, Alcantara, \& Weisblatt, 2003; O'Riordan, Plaisted, Driver, \& Baron-Cohen, 2001). In such tasks, subjects are required to detect a target in a display containing a variable number of distracters. If the task difficulty is increased by adding distracters, usually RTs increase. In studies on PDD, two search task versions that differed in difficulty have been used. In both tasks children with PDD showed shorter RTs, as compared with normally developing controls (Plaisted et al., 1998; Plaisted et al., 2003; O'Riordan et al., 2001). The reason for this superior performance is not clear, but has been related to enhanced ability to discriminate between stimulus elements in subjects with PDD (O'Riordan \& Plaisted, 2001), an explanation that has also been proposed for the superior performance of subjects with autism on other visuo-spatial tasks (Plaisted et al., 2003, see also Bertone, Mottron, Jelenic, \& Faubert, 2001).

So far, however, there have been no studies that have validated the claim for enhanced stimulus discrimination as an explanation for superior performance in search tasks in PDD more directly. It is well-known that stimulus discriminability affects eye movement parameters, especially number of fixations and fixation duration (Hooge \& Erkelens, 1999). Therefore, studying eye movements during search tasks in subjects with PDD is a first step to gain 
more insight into the neurocognitive mechanisms of atypical visuo-spatial processing in this group. If increased stimulus discriminability is indeed the underlying factor for superior performance of subjects with PDD, it is expected that they need fewer and/or shorter fixations to identify the target. Also, eye movement parameters can be used to test alternative hypotheses for shorter reaction times in search tasks in PDD. More specifically, it has been argued that (healthy) subjects in a visual search task have a tendency to move their eyes even though in some situations it would be a better strategy not to do so, since longer fixations allow better extraction of (peripheral) information (Hooge \& Erkelens, 1999; see also Rayner, 1998). It is well possible that individuals with PDD use a different search strategy in which they show longer, but less, fixations than controls.

The first aim of the present study was to use eye movement parameters to test the two hypotheses described above for superior visual search in subjects with PDD. A second aim was to replicate the findings of Plaisted et al. (1998) and O'Riordan and Plaisted (2001) in high functioning adults with PDD. For these reasons we used the same search tasks as described in O'Riordan et al. (2001, second experiment) in a group of high functioning adults with PDD, matched on gender, IQ, and age to a control group.

\section{Methods}

\section{Participants}

Two groups of young male adults participated, a group of eight high functioning individuals with PDD and a group of eight normal control individuals. The clinical subjects were recruited via the Department of Child and Adolescent Psychiatry at the Utrecht University Hospital. The study was described to the subjects and written informed consent was obtained. All subjects were administered the Wechsler Intelligence Scale, revised Dutch edition (WAIS). Diagnoses of either Autistic Disorder or Asperger Syndrome were made by a child psychiatrist and based on DSM-IV criteria. The parents of all subjects with PDD were administered the Autism Diagnostic Interview Revised (ADI-R) (Lord, Pickles, McLennan \& Rutter, 1997; Lord, Rutter \& LeCouteur, 1994). Due to technical problems, eye-movement data of one subject with PDD and one control subject were lost. The individual ADI scores of the remaining seven PDD subjects can be found in Table 1 . Unpaired $t$-tests revealed that there were no significant differences between the remaining seven PDD and seven control subjects with respect to either age, TIQ, VIQ, or PIQ (see Table 2). None of the participants showed any visual or oculomotor pathology other than refraction
Table 1 ADI scores on the four domains for individual PDD subjects

\begin{tabular}{lllll}
\hline $\begin{array}{l}\text { ADI scores } \\
\text { individual } \\
\text { subjects }\end{array}$ & $\begin{array}{l}\text { Social } \\
\text { interaction } \\
\text { (cutoff 10) }\end{array}$ & $\begin{array}{l}\text { Communication } \\
\text { (cutoff 8) }\end{array}$ & $\begin{array}{l}\text { Stereotypy } \\
\text { (cutoff 3) }\end{array}$ & $\begin{array}{l}\text { Age of } \\
\text { onset } \\
\text { (cutoff 1) }\end{array}$ \\
\hline 1 & 22 & 18 & 2 & 0 \\
2 & 18 & 13 & 9 & 0 \\
3 & 24 & 16 & 3 & 5 \\
4 & 26 & 16 & 8 & 5 \\
5 & 24 & 24 & 10 & 3 \\
6 & 21 & 20 & 4 & 3 \\
7 & 28 & 17 & 5 & 2 \\
\hline
\end{tabular}

Table 2 Mean age and IQ characteristics of the control and PDD group

\begin{tabular}{llr}
\hline & Control & PDD \\
\hline Age & 21.2 & 22.1 \\
TIQ & 115.3 & 121.9 \\
VIQ & 119.1 & 124.0 \\
PIQ & 106.7 & 114.0 \\
\hline
\end{tabular}

anomalies. Subjects were allowed to wear their contact lenses or glasses.

Set up and Data Analysis

Subjects sat in front of a LaCie Blue Electron $11122^{\prime}$ Screen $(0.394 \mathrm{~m} \times 0.295 \mathrm{~m}, 1240 \times 1024$ pixels at $85 \mathrm{~Hz}$,$) at a$ distance of $0.64 \mathrm{~m}$. Stimuli were generated by a Apple PowerMac G4/450 DP using a Matlab program based on routines taken from the Psychophysics Toolbox (Brainard, 1997; Pelli, 1997) and the EyeLink Toolbox (Cornelissen, Peters \& Palmer, 2002).

Movements of the left eye were measured at $250 \mathrm{~Hz}$ with the EyeLink 1 eye monitor. Head movements were prevented by the use of a chinrest. Data were stored on disk and were analysed off-line by a self-written Matlab program. The velocity signal of eye movements was searched for peak velocities above $20 \%$ s. Each peak (in the velocity signal) was considered a potential indicator of the presence of a saccade. The exact onset of the saccade was determined by going backward in time to the point where the velocity signal dropped below the average velocity plus two standard deviations during the stable fixation period before the saccade. The exact offset of the saccade was determined by going forward in time to the point where the velocity signal dropped below the average velocity plus two standard deviations during the stable fixation period after the saccade. This method was adopted from Van der Steen and Bruno (1995). This procedure was followed by 
rejection/acceptation based on minimum saccade duration of $12 \mathrm{~ms}$ and minimum amplitude of $1^{\circ}$. When a saccade was removed, fixation time before and after this saccade and the duration of the saccade were added together.

Stimulus and Task

Two tasks were tested in separate sessions for each participant, with the order counterbalanced within participant groups. Both tasks contained tilted as well as vertical line elements. The tasks only differed in which of the two elements was target or distracter. Since an earlier study has shown a difference in task difficulty depending on target orientation (O'Riordan et al., 2001), the condition including a vertical target among tilted distracters will be referred to as the easy condition, and the condition with a tilted target (among vertical distracters) as the hard condition. Each task contained two crossed factors; set size $(4,16$, or 25 items) and probe, target present or target absent. There were 30 trials at each unique combination of factors, yielding a session of 180 trials per task. The sequence of different tasks was randomised within each session. In each session, the participant was informed about the nature of the target (either vertical or tilted), but did not know when a target would be present or absent, or what the display size would be on any trial. The participants performed a binary choice RT task indicating whether a target was present or absent by a button press on each trial.

\section{Procedure}

The order in which the two conditions were presented was counterbalanced. Prior to each task participants were given 10 practice trials. Participants were instructed to respond as quickly and accurate as possible by pressing the five key on the numeric pad when the target was present and the four key when the target was absent. Thereafter, the eye link camera was attached to the head and calibrated. On each trial the sequence of events was as follows: a fixation cross was presented. The participant had to fixate at the cross and press the space bar subsequently. Fixation of the fixation cross was used for on-line drift correction of the eye tracker. Then, the search display appeared and remained visible until the participant responded. After the response the next trial started.

\section{Results}

To assure that a possible superior task performance of the individuals with PDD was not due to a difference in detection criteria (e.g., if speeded reaction time is accompanied by a decrease in number of correct answers), we performed an independent $t$-test on the error rates of both groups. No significant difference in error rate between the groups was found. One of the subjects in the PDD group showed a high number of errors and a deviant pattern in reaction time data, and was excluded from further analysis. The results described below are therefore based on six subjects with PDD, and seven controls.

The mean RT, first fixation times, remaining fixation times, and number of fixations data were analysed using a repeated measures ANOVA, with one between subjects factor of group (control or autistic) and three within subject factors, condition (condition 1: tilted target or condition 2: vertical target), probe (target present or target absent) and set size $(4,16$, or 25 items). Analyses of the first and remaining fixations were done separately, because there is evidence that they belong to different distributions (Hooge \& Erkelens, 1996). Unless otherwise stated, a significance level of $p<.05$ was adopted for all statistical comparisons. Only main effects or interactions with Group will be considered.

\section{RT Analysis}

The analysis of variance revealed a main effect of Group, $F(1,11)=6.6, p<0.05$, reflecting the fact that individuals with PDD were significantly faster overall than the control individuals (737 vs. $1016 \mathrm{~ms})$. A significant interaction Group $\times$ Display was found $(F(2,22)=3.8, p<.05)$, as well as an interaction of Group $\times$ Display $\times$ Probe $(F(2$, $22)=4.7, p<.05)$. Partial analyses showed that the Groups difference was significant at all set sizes, but that the effects were largest for set size 25 (all $p<.05$ ) (resp., $F(1,11) 5.7,5.4,6.8)$. The interaction Group $\times$ Probe was marginally significant for set size 25 only $(F(1,11)=4.0$, $p=0.72$ ), indicating that at this set size, the Groups difference was largest if the target was absent (See Fig. 1).

\section{Eye Movement Analysis}

The analysis of number of fixations revealed a significant effect of group $(F(1,11)=10.3, p<.01)$. The individuals with PDD made significantly less fixations than the control group (means, respectively, 1.3 and 2.1). In addition, there were significant interactions between Group $\times$ Probe, Group $\times$ Display and Group $\times$ Probe $\times$ Display. When tested per level of Display, subjects with PDD showed significantly fewer fixations for all display sizes $(F(1,11)=8.2$, $p=.015 / 9.4, p=.011 / 10.8, p=.007)$, but the effects were largest for the 25 set size. Interactions of Group $\times$ Probe effects were marginally significant for all set sizes $(F(1,11)=4.7, p=.052 / 4.0, p=.072 / 4.4, p=.06)$, indicating that group differences in number of fixations were largest in the target absent condition (see Fig. 2). 
Fig. 1 Means and standard errors of the reaction times for each group in trials with (left panel) and without a target (right panel), for the easy and hard condition
Fig. 2 Means and standard errors of the number of fixations for each group in trials with (left panel) and without a target (right panel), for the easy and hard condition
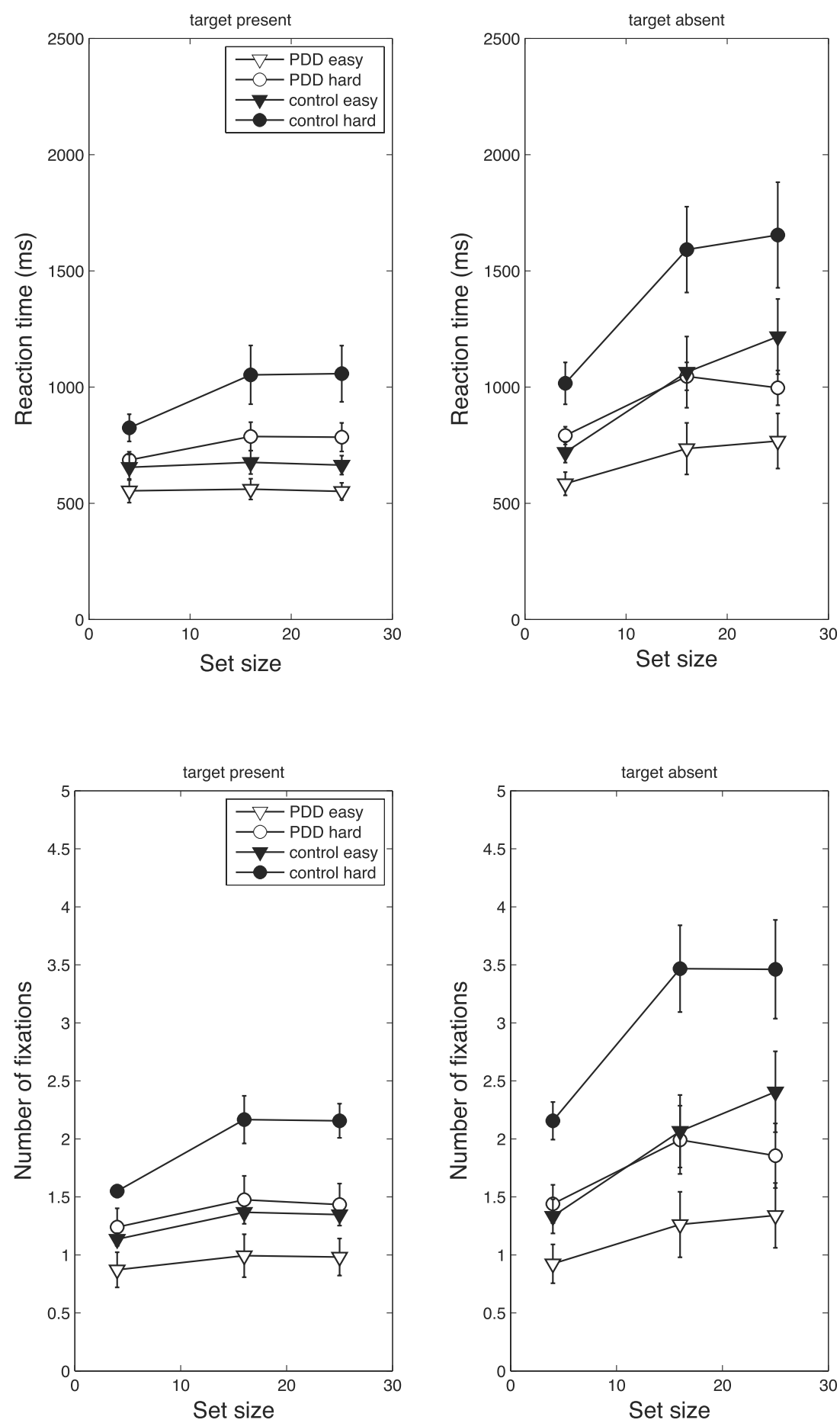

No significant group differences were found for fixation times at either the first fixation or the remaining fixations.

\section{Discussion}

The present study was aimed to test two hypotheses on superior performance in visual search tasks in subjects with
PDD. It has been suggested earlier that this superior performance reflects an enhanced ability to discriminate between stimulus elements (O'Riordan et al., 2001). We tested this hypothesis by measuring eye movements during search tasks in high functioning adult men with PDD and an age- and IQ-matched control group. In case of enhanced stimulus discriminability, shorter fixation times and/or fewer fixations were expected in the PDD group, as com- 
pared to the control group. The alternative explanation, that subjects with PDD use more efficient search strategies, was also tested. A more efficient search strategy would be reflected in a longer fixation time.

The reaction time data showed that subjects with PDD were better than the control group for both easy and hard search tasks, especially in the largest set size and in trials in which the target was absent. These findings are in accordance with earlier studies on visual search in children with autism, indicating faster RT in the same type of tasks (O'Riordan et al., 2001; Plaisted et al., 1998). This indicates that superior search performance in subjects with PDD is a robust finding that can be demonstrated in both children and adults.

Analyses of fixations during the search tasks showed that subjects with PDD made fewer fixations than controls. This difference in number of fixations was especially clear for the largest set size and for the condition in which the target was absent, analogous to the RT effects. In addition, it was noted that many subjects with PDD showed an absence of saccadic eye movements in target present trials, indicating that they were able to localize the target in a single glance. No differences between groups were found in fixation time. The absence of group differences in fixation time indicates that subjects with PDD followed the same search strategy as normal controls. However, the decreased number of fixations during the task in the PDD group is in agreement with the hypothesis of enhanced stimulus discriminability in subjects with PDD, as suggested by O'Riordan and Plaisted (2001). The finding that the pattern of effects for fixation times was strongly similar to the RT effect provides additional evidence that superior performance in this task is indeed related to enhanced discriminatory abilities in PDD.

The question what the locus of this enhanced discriminability could be has been addressed by Cohen (1994), who tested a neural network model of processing in subjects with PDD. This model showed that an increase in processing units resulted in a strong ability to discriminate and learn stimulus patterns, along with a weak ability for generalization. Indeed, there is anatomical evidence for abnormal development of the cyto-architecture of the cerebral cortex of subjects with PDD, resulting in an increase in processing units. Recent post-mortem studies in subjects with PDD show an increased number of micro columns, albeit of reduced size (Casanova, Buxhoeveden, Switala, \& Roy, 2002; Casanova et al., 2006).

Concluding, the measurement of eye movements during search tasks shows that the superior behavior of subjects with PDD in these tasks cannot be attributed to strategy difference, but point indeed to increased stimulus discriminability in this group.
Acknowledgments The work described was supported by an Innivational Research Incentives grant (VIDI-scheme, 402-01-094) of the Netherlands Organisation for Scientific Research (NWO) to Chantal Kemner. We are grateful to the assistence of Renate Simons in recruitment of the subjects.

\section{References}

Bertone, A., Mottron, L., Jelenic, P., \& Faubert, J. (2005). Enhanced and diminished visuo-spatial information processing in autism depends on stimulus complexity. Brain, 128, 2430-2441.

Brainard, D. H. (1997). The psychophysics Toolbox. Spatial Vision, $10,433-436$.

Casanova, M. F., Buxhoeveden, D. P., Switala, A. E., \& Roy, E. (2002). Minicolumnar pathology in autism. Neurology, 58, 428-432.

Casanova, M. F., van Kooten, I. A., Switala, L. A. E., van Engeland, H., Heinsen, H., Steinbusch, H. W., Hof, P. R., Trippe, J., Stone, J., \& Schmitz, C. (2006). Minicolumnar abnormalities in autism. Acta Neuropathologica (Berl), 112, 287-303.

Cohen, I. L. (1994). An artificial neural network analogue of learning in autism. Biological Psychiatry, 36, 5-20.

Cornelissen, F. W., Peters. E., \& Palmer, J. (2002). The Eyelink Toolbox: Eye tracking with MATLAB and the Psychophysics Toolbox. Behavior Research Methods, Instruments \& Computers, 34, 613-617.

Dakin, S., \& Frith, U. (2005). Vageries of visual perception in autism. Neuron, 48, 497-507.

Hooge, I. T., \& Erkelens, C. J. (1996). Control of fixation duration in a simple search task. Perception and Psychophysics, 58, 969-976.

Hooge, I. T., \& Erkelens, C. J. (1999). Peripheral vision and oculomotor control during visual search. Vision Research, 39, $1567-1575$.

Lord, C., Rutter, M., \& LeCouteur, A. (1994). Autism diagnostic interview-Revised: A revised version of a diagnostic interview for caregivers of individuals with possible pervasive developmental disorders. Journal of Autism and Developmental Disorders, 24, 659-685.

Lord, C., Pickles, A., McLennan, J., \& Rutter, M. (1997). Diagnosting autism: Analyses of data from the autism diagnostic interview. Journal of Autism and Developmental Disorders, 27, 501-517.

O'Riordan, M, Plaisted, K., Driver, J., \& Baron-Cohen, S. (2001). Superior visual search in autism. Journal of Experimental Psychology: Human Perception and Performance, 27, 719-730.

O'Riordan, M. A., \& Plaisted, K. C. (2001). Enhanced discrimination in autism. Quarterly Journal of Experimental Psychology A, 54, 961-979.

Pelli, D. G. (1997). The VideoToolbox software for visual psychophysics: Transforming numbers into movies. Spatial Vision, 10, $437-442$.

Plaisted, K., O’Riordan, M., \& Baron-Cohen, S. (1998). Enhanced visual search for a conjunctive target in autism: a research note. Journal of Child Psychology and Psychiatry, 39, 776-783.

Plaisted, K., Saksida, L., Alcantara, J., \& Weisblatt, E. (2003). Towards an understanding of the mechanisms of weak central coherence effects: Experiments in visual configural learning and auditory perception. Philosophical Transactions of the Royal Society of London, B, 358, 375-386.

Rayner, K. (1998). Eye movements in reading and information processing: 20 years of research. Psychology Bulletin, 124, 372422.

Van der Steen, J., \& Bruno, P. (1995). Unequal amplitude saccades produced by aniseikonic patterns: Effects of viewing distance. Vision Research, 35, 3459-3471. 